\title{
Dementia care management: going new ways in ambulant dementia care within a GP-based randomized controlled intervention trial
}

\author{
Tilly Eichler, ${ }^{1}$ Jochen René Thyrian, ${ }^{1}$ Adina Dreier, ${ }^{2}$ Diana Wucherer, ${ }^{1}$ Leonore Köhler, ${ }^{1}$ \\ Thomas Fiß, ${ }^{1}$ Ceorgia Böwing, ${ }^{1}$ Bernhard Michalowsky ${ }^{1}$ and Wolfgang Hoffmann ${ }^{1,2}$ \\ ${ }^{1}$ German Center for Neurodegenerative Diseases (DZNE) Rostock/Greifswald, Germany \\ ${ }^{2}$ Section Epidemiology of Health Care and Community Health, Institute for Community Medicine, Ernst-Moritz-Arndt-University, Greifswald, Germany
}

Background: Increasing prevalence of dementia is a major challenge for the German healthcare system. The study DelpHi-MV ("Dementia: life- and person-centered help in Mecklenburg-Western Pomerania") aims to implement and evaluate an innovative subsidiary support system for persons with dementia (PwDs) living at home and their caregivers.

Methods: DelpHi-MV is a GP-based cluster randomized controlled intervention trial. DelpHi-Intervention aims to provide "optimum care" by integrating multi-professional and multimodal strategies to individualize and optimize treatment of dementia within the framework of the established healthcare and social service system. The intervention is conducted by Dementia Care Managers (DCMs) - nurses with dementia-specific training - at the people's homes. Based on German guidelines for evidence-based diagnoses and treatment of dementia, a comprehensive set of 95 intervention modules - the "DelpHi-Standard" - was defined. Each module consists of predefined trigger condition(s), a subsequent intervention task, as well as at least one criterion for its completion. The intervention begins with a comprehensive assessment of the care situation, needs, and resources. The DCM develops and implements an intervention plan tailored to the individual conditions in close cooperation with the GP.

Expected Results and Conclusions: We expect statistically significant differences between control and intervention group in primary outcomes (quality of life, behavioral and psychological symptoms of dementia, pharmacotherapy, caregiver burden). Results can provide evidence for the effectiveness and efficacy of dementia care management according to the "Delphi-Standard" - prerequisites for implementing this concept into routine healthcare.

Key words: dementia care management, integrative, intervention, GP-based

\section{Introduction}

Aging society coupled with increasing incidence and prevalence of dementia (Fendrich and Hoffmann, 2007; Hampel et al., 2011) is a major challenge for the German healthcare system. Since causal treatment of dementia is still out of sight, there is a considerable need for new and more effective ways of dementia care to address the impending healthcare crisis (Naylor et al., 2012).

The complexity of dementia demands comprehensive individualized care that addresses physical, psychological, social, and legal issues of the persons

Correspondence should be addressed to: Dr. Tilly Eichler, German Center for Neurodegenerative Diseases (DZNE) Rostock/Greifswald, Ellernholzstrasse 1-2, Greifswald D-17487, Germany. Phone: +49-(0)-3834-86-7591; Fax: +49-(0)-3834-86-19551. Email: tilly.eichler@dzne.de. Received 8 Mar 2013; revision requested 10 Apr 2013; revised version received 25 Apr 2013; accepted 18 Sep 2013. First published online 23 October 2013. with dementia (PwDs) as well as their caregivers. The established healthcare system in Germany offers a broad range of healthcare services such as GPs, neurologists, psychiatrists, pharmacists, geriatric rehabilitation, qualified nursing care with diverse ambulatory services and in- and outpatient facilities, self-help groups, or caregiver counseling. However, fragmentation of these services as well as deficient communication and integration between them is a major problem and as a result utilization depends too often on individual, regional, logistic, or structural variables (Reuben et al., 2009; Thyrian et al., 2012). Therefore, integration and coordination rather than duplication of established services seems to be the method of choice to optimize dementia care in the ambulant sector.

As the majority of PwDs are treated by their GP, the primary care sector plays a key role in optimizing 
dementia care (Wagner and Abholz, 2002). Recent studies show the effectiveness of collaborative care for PwDs in the primary care setting (Callahan et al., 2006; Vickrey et al., 2006). A review of six RCTs with case management programs for PwDs indicates that case management intensity and the integration level between health and social service organizations relate to greater efficacy (Somme et al., 2012). However, since all, but one, studies were conducted in the USA, it remains unclear whether results can be readily generalized to the healthcare system in Germany.

Most PwDs wish to remain in their homes as long as possible. To allow adequate home care the support by an informal caregiver is usually required, particularly in later stages of dementia. However, caregivers carry a high burden. Studies show considerable impact on their physical and mental health (Black and Almeida, 2004), often associated with earlier institutionalization of the PwDs (Mittelman et al., 2006; Etters et al., 2008). Hence, adequate support of caregivers is an indispensable part of any dementia care management.

With DelpHi-MV ("Dementia: life- and personcentered help in Mecklenburg-Western Pomerania") we want to implement and evaluate an innovative GP-based subsidiary support system for PwDs living at home and their caregivers. The DelpHi-Intervention is designed (1) to integrate multi-professional and multimodal strategies to optimize and individualize comprehensive treatment of dementia within the framework of the established healthcare and social service systems, (2) to diagnose cognitive impairment at an early stage to allow for early initiation of therapy as well as to take all necessary steps to prepare for progression of disease, (3) to address multimorbidity and its treatment that could interfere with dementia care or aggravate the course of dementia, and (4) to adequately address all relevant dimensions of caregiver burden.

Primary outcome measures of the intervention are: (1) improvement of quality of life ( $\mathrm{PwD}$, caregiver), (2) reduction of behavioral and psychological symptoms of dementia, (3) optimizing pharmacotherapy, and (4) reduction of caregiver burden (Thyrian et al., 2012).

Purpose of this paper is to provide a comprehensive description of the DelpHi-Intervention.

\section{Methods}

\section{Design, setting, participants}

DelpHi-MV is a population-based clusterrandomized controlled intervention trial in the primary care setting; details (including sample size calculation) are described in the study protocol (Thyrian et al., 2012). Enrolment into the study started in January 2012; the estimated primary completion date (first follow-up) is January 2015. Eligible patients (older than 70 years, living at home) of participating GP-practices are screened for cognitive impairment with the DemTect (Calabrese and Kessler, 2000). Patients who meet the inclusion criteria (DemTect $<9$ ) are informed by their GP about the study and are asked to participate and to sign a written consent. If the patient can name a caregiver, he or she is asked to participate as well. In case the patient is not able to give informed consent, his or her legal representative is asked to sign (as approved by the Ethical Committee of the Chamber of Physicians of Mecklenburg-Western Pomerania, registry number BB 20/11).

Participants and their caregivers are clusterrandomized into an intervention and a control group. Participating GP-practices in MecklenburgWestern Pomerania represent the clusters for randomization (1:1 allocation ratio). Baseline and annual follow-up assessments are conducted identically in both groups. Whereas the control group receives "care as usual," the intervention group receives the "DelpHi-Intervention" (see Figure 1 for an outline of the DelpHi-Intervention). The intervention is initiated and coordinated by Dementia Care Managers (DCMs), qualified nurses who received a dementia-specific training (Dreier et al., 2011).

\section{Baseline assessment}

Patients who were included into the study are contacted by their designated DCM to arrange two to three personal visits at their homes to carry out a comprehensive standardized baseline assessment. The DCM collects standardized data regarding each patient's specific social, medical, psychological, pharmaceutical, and nursing situation to identify in detail the individual needs and resources of the $\mathrm{PwD}$ and his or her caregiver (for details see Table 1 ).

The baseline assessment includes a home medication review (HMR; Fiß et al., 2013; submitted). The DCM records all drugs used by the PwD, including non-prescription medicines. In addition the DCM collects data about storage, usage (dosage, time to meal), adherence, adverse drug events, and other drug-related problems. For the intervention group this information is summarized and passed on to the regular pharmacy of the PwD, provided the PwD consented in forwarding this data and the specified pharmacy 


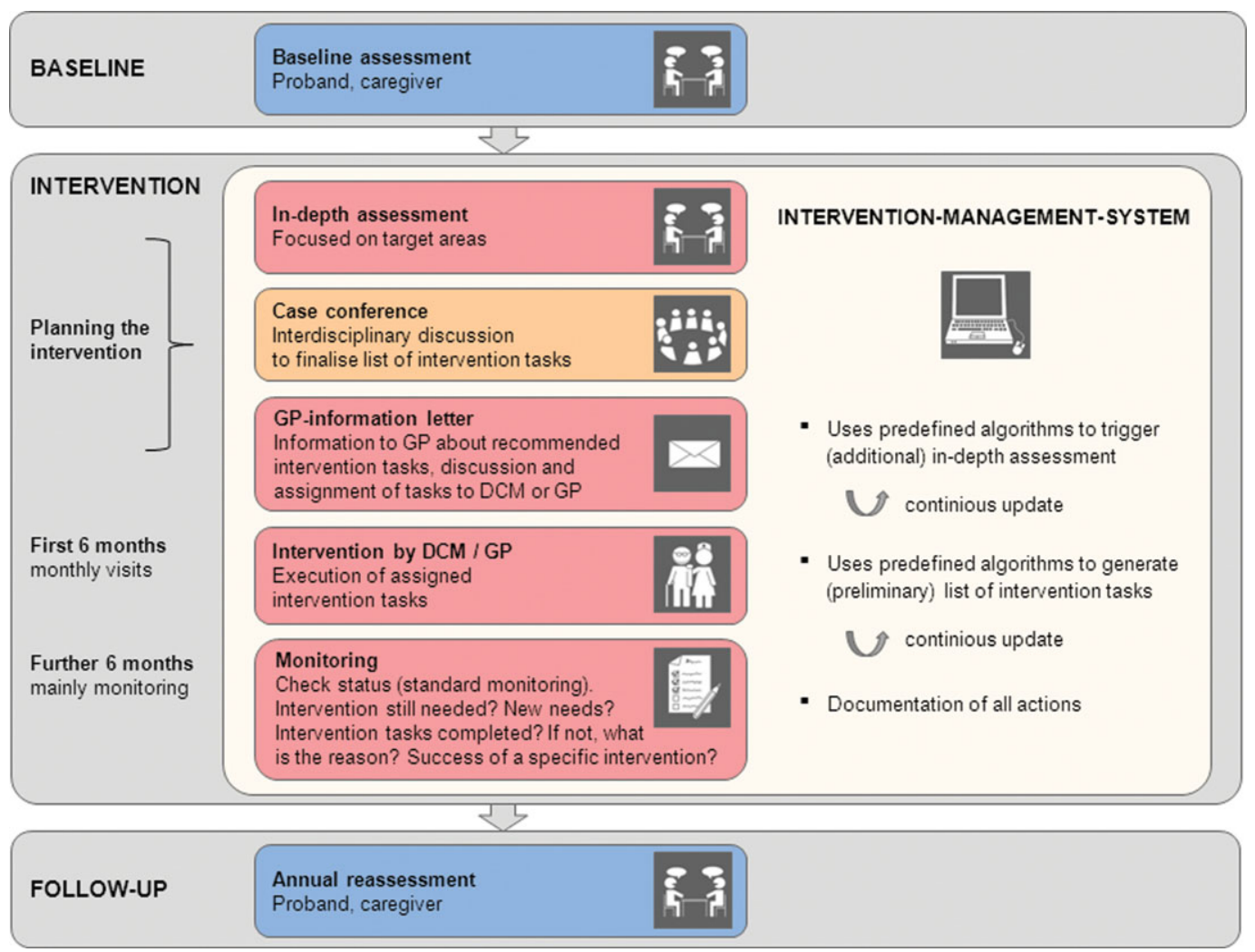

Figure 1. Outline of DelpHi-Intervention.

participates in the DelpHi-MV study as well. The pharmacist is trained to conduct the standardized DelpHi-medication review concerning drug-related problems such as double prescriptions, side effects, interactions, or potentially inappropriate medications that carry an increased risk of adverse drug events in elderly patients according to the German PRISCUS-list (Holt et al., 2010). The resulting medication review including specific pharmaceutical advices is faxed to the study center. The DCM includes the medication review and the pharmacist's advice in her GP-information letter (see below). In case the regular pharmacy of a PwD is not participating, the DelpHi-MV study pharmacist conducts the medication review instead.

\section{Intervention: the "DelpHi-Standard"}

After baseline assessment the intervention group receives the DelpHi-Intervention according to the "DelpHi-Standard" for one year. The DelpHiStandard was designed to optimize care for the PwDs and their caregivers according to the World Health Organization (1986) definition of health: "Health is a state of complete physical, mental and social well-being and not merely the absence of disease or infirmity." To meet this requirement, the intervention has to be complex, multi-professional, multimodal, and adjusted to individual needs and resources of the individual PwD and his or her caregiver as well as to local conditions.

The development of the DelpHi-Intervention is based on German guidelines for evidence-based diagnostic and treatment of dementia (DEGAM, 2008; DGPPN and DGN, 2009), a review of current literature (e.g. Vollmar et al., 2007; Förstl, 2009), meetings and symposia with experts in the field, and the scientific advisory board of DelpHiMV. To describe the "DelpHi-Standard," three key areas - the "pillars of intervention" - where defined: (1) management of treatment and care, (2) medication management, and (3) caregiver support and education. Associated with these pillars are eight different action fields, where each action field includes several foci. Every focus comprises specific intervention modules that constitute the actual basis for the work of the DCM (see Figure 2).

Each intervention module consists of defined algorithms that contain the trigger condition(s) derived from standardized baseline and/or in-depth assessment (see Table 1) that activate(s) a specific intervention task and at least one criterion for successful completion of that task (see Figure 3).

The DelpHi-Intervention comprises 95 of such predefined intervention modules. Feasibility, adequacy, and acceptance of intervention modules were tested and revised during the pilot phase of the study $(n=6 \mathrm{GPs}, n=21$ probands of 
Table 1. Instruments used at baseline and in-depth assessment

ASSESSMENT TYPE

ASSESSMENT OF:

Baseline assessment

Sociodemographic characteristics/social environment (age, marital status, children, household members, education, income, last professional position, living situation, persons who can take care on short notice): SDQ

Informations about PwD (cognitive capacity for consent, power of attorney, Patient Decree, legal representative, Pflegestufe (care level), disabled pass, etc.) $\mathrm{SDQ}^{3}$

Utilization of healthcare resources/social services/nursing aids: $\mathrm{SDQ}^{2,3}$

General state of health: Extract from Standardized Assessment of Elderly People in primary care $(\text { STEP) })^{2,3}$ (Sandholzer et al., 2004)

Health-related quality of life: 12-Item Short Form Health Survey (SF-12) ${ }^{2}$ (Bullinger and Kirchberger, 1998)

Activities of daily livings: Bayer Activities of Daily Living Scale (B-ADL) ${ }^{2,3}$ (Hindmarch et al., 1998)

Knowledge about dementia, treatment options, and healthcare/social services: $\mathrm{SDQ}^{3}$

Social support: Fragebogen zur sozialen Unterstützung (F-SozU) ${ }^{2,3}$ (Fydrich et al., 2007)

Quality of life: Quality of Life - Alzeimer's Disease (Qol-AD) ${ }^{1}$ (Logsdon et al., 2002)

Depression: Geriatric Depression Scale (GDS) ${ }^{2,3}$ (Reisberg et al., 1988)

Cognitive impairment/severity grading of cognitive dysfunction: Structured Interview for the

Diagnosis of Dementia (SIDAM/SISCO) ${ }^{2,3}$ (Zaudig et al., 1991)

Medication (intake, drug-related problems, potentially inadequate medications, adherence):

Home Medication Review (HMR) ${ }^{1,2,3}$ (Fiß et al., 2013; submitted)

Comorbidities (hypertension, diabetes, stroke): $\mathrm{SDQ}^{2}$

Psychiatric symptoms: Neuropsychiatric Inventory (NPI) ${ }^{1,3}$ (Cummings, 1997)

Medical status (ICD-10 diagnosis, hospitalization): Patient record of $\mathrm{GP}^{2,3}$

Informal care: Resource Utilization in Dementia (RUD) ${ }^{2}$ (Wimo et al., 1998)

Caregiver burden: Berliner Inventar zur Angehörigenbelastung Demenz (BIZA-D) ${ }^{1,3}$ (Zank et al., 2006); Brief Symptom Inventory (BSI) ${ }^{2}$ (Derogatis and Melisaratos, 1983); Patient Health

Questionnaire (PHQ-D) ${ }^{2}$ (Löwe et al., 2002)

\section{In-depth assessment (Intervention)}

Standard assessment conducted at each intervention visit (emergencies, state of health, medical consultations, hospitalization, changes in medication, falls, pain, change in cognitive impairment, liquid intake, blood pressure, blood sugar): $\mathrm{SDQ}^{3}$

Neurological or psychiatric symptoms: $\mathrm{SDQ}^{3}$

Suitability for rehabilitation: $\mathrm{SDQ}^{3}$

Restriction in mobility: Timed up \& go ${ }^{3}$ (Podsiadlo and Richardson, 1991)

Risk of falling: Tinetti-Test ${ }^{3}$ (Tinetti, 1986)

Cognitive performance: Mini-Mental State Examination (MMSE) ${ }^{3}$ (Kessler et al., 1990)

Depression: Mini International Neuropsychiatric Interview (M.I.N.I. Depression) ${ }^{3}$ (Lecrubier $^{2}$ et al., 1997)

Pain assessment: Beurteilung von Schmerzen bei Demenz (BESD) ${ }^{3}$ (Basler et al., 2006)

Clinical Dementia Rating (CDR) ${ }^{3}$ (Berg, 1988)

Severity of dementia: $\mathrm{SDQ}^{3}$

Care Needs Assessment for Dementia (CareNapD) ${ }^{3}$ (McWalter et al., 1998)

Caregiver burden: Healthy Age Brain Monitor (HABC) ${ }^{3}$ (Monahan et al., 2012)

PwD, C

PwD, C

PwD, C

PwD, C

PwD

PwD

PwD

PwD

PwD

PwD

PwD

PwD

$\mathrm{C} \rightarrow \mathrm{PwD}$

$\mathrm{GP} \rightarrow \mathrm{PwD}$

C

C

PwD

PwD

${ }^{1}$ Primary outcome measure.

${ }^{2}$ Secondary outcome measure.

${ }^{3}$ Trigger for intervention task.

Note: C, caregiver; DCM, Dementia Care Manager; PwD, person with dementia; C/DCM $\rightarrow$ PwD, assessment of PwD by C or DCM; SDQ, self-developed questionnaire.

intervention group). Since the study takes place in the setting of routine care new developments in treatment guidelines will be incorporated into the intervention, otherwise the intervention will not be modified while it is being tested.
The comprehensive list of intervention modules helps to ensure that (1) all PwDs and their caregivers receive tailored interventions according to their individual needs and resources, and (2) the DCM would not overlook any necessary aspects of 


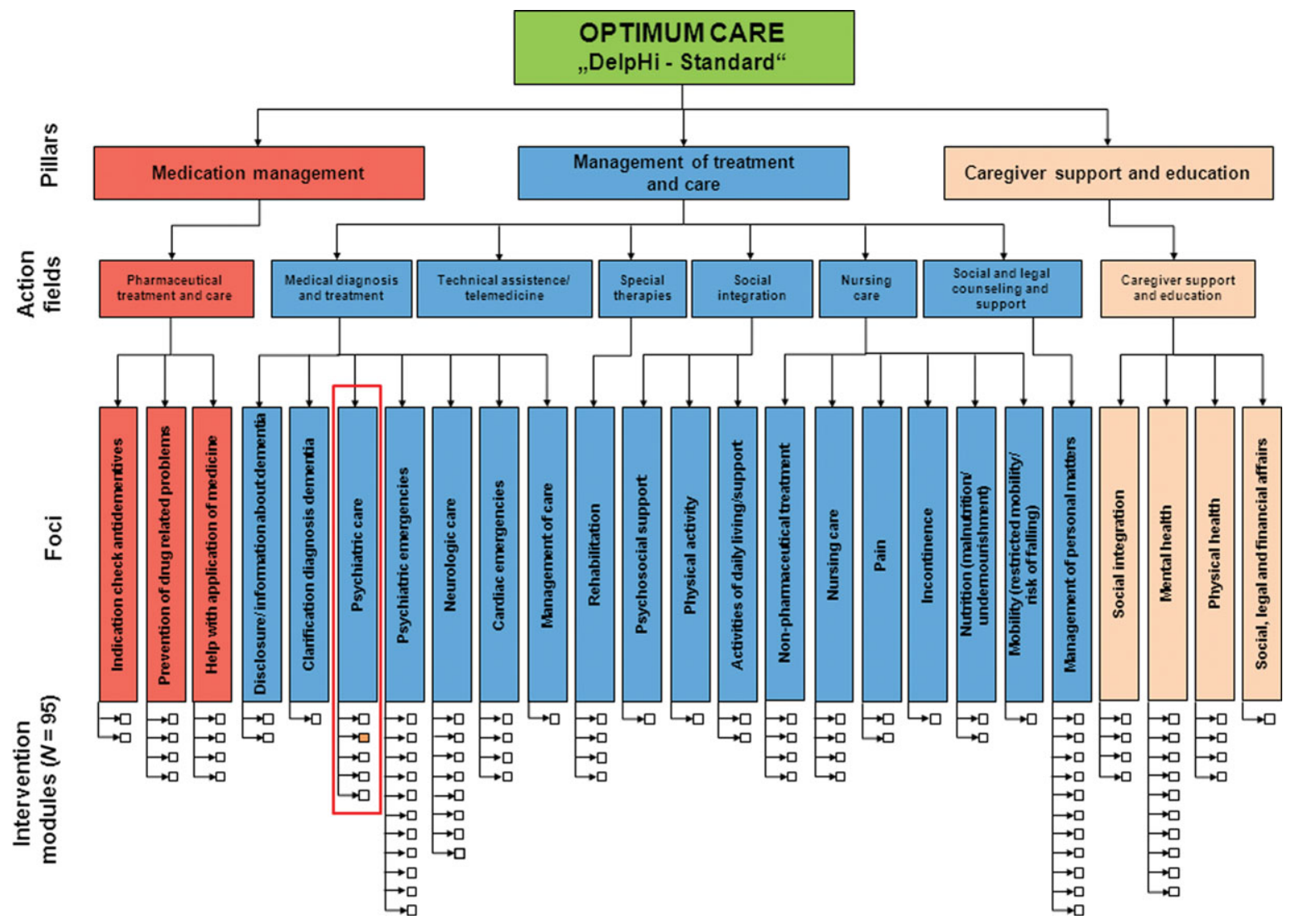

Figure 2. Optimum Care as "DelpHi-Standard": pillars, action fields, foci, and intervention modules (red marked area see Figure 3).

Focus INTERVENTION MODULES

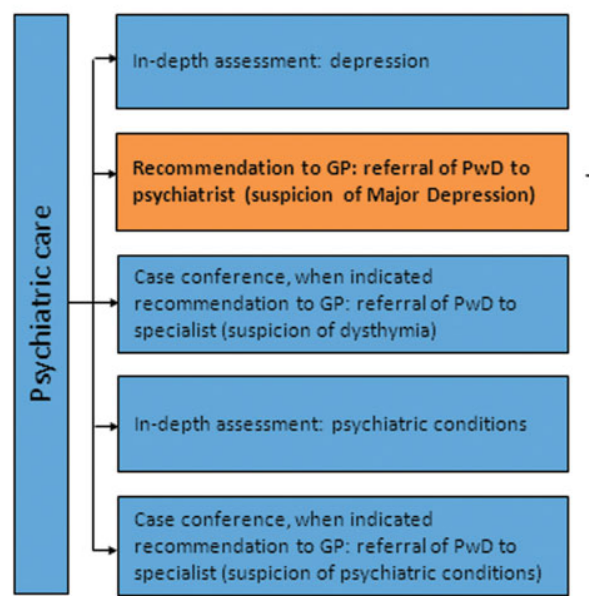

Figure 3. Example of an intervention module.

DelpHi-Intervention despite its broad spectrum and complexity.

There are following different types of intervention modules, examples of which are shown in Table 2:

(1) In-depth assessment: standard assessment indicated a specific problem; the DCM needs to do further in-depth assessment to plan further intervention
(2) Case conference: assessment indicated a problem that needs to be discussed in an interdisciplinary case conference to decide whether a specific intervention task is indicated and should be recommended to the GP

(3) Evaluation of the situation by DCM: some situations are too complex to address with predefined algorithms; the DCM has to assess the individual situation and decide whether specific intervention tasks are indicated 
Table 2. Examples for different types of intervention modules

\begin{tabular}{|c|c|c|c|c|c|}
\hline INT & ERVENTION MODULE & $\begin{array}{l}\text { IF } \\
\text { (TRIGGER CONDITION) }\end{array}$ & $\begin{array}{l}\text { THEN } \\
\text { (INTERVENTION TASK) }\end{array}$ & $\begin{array}{l}\text { WHO IS } \\
\text { RESPONSIBLE? }\end{array}$ & $\begin{array}{l}\text { MONITORING: CRITERIA FOR } \\
\text { COMPLETION OF TASK }\end{array}$ \\
\hline (1) & $\begin{array}{l}\text { In-depth assessment: } \\
\text { Depression }\end{array}$ & $\begin{array}{l}\text { GDS }>5 \text { (mild to severe depression) } \\
\text { AND/OR NPI-16: Depression } \\
\text { (scale D): frequency } \geq 3 \text { AND } \\
\text { severity } \geq 1 \text { AND/OR standard } \\
\text { monitoring: clinical impression of } \\
\text { DCM: "depressive" }\end{array}$ & Conduct M.I.N.I. Depression & DCM & M.I.N.I. Depression conducted \\
\hline (2) & $\begin{array}{l}\text { Case conference: } \\
\text { Neurological } \\
\text { symptoms }\end{array}$ & $\begin{array}{l}\text { Semi-paralysis OR facial paralysis } \\
\text { ("not acute") AND/OR dizziness } \\
\text { AND/OR aphasia ("mild" OR } \\
\text { "moderate") }\end{array}$ & $\begin{array}{l}\text { Discussion of neurologic symptoms } \\
\text { in case conference, if indicated } \\
\text { recommendation to GP: referral } \\
\text { to neurologist }\end{array}$ & $\mathrm{DCM}(\rightarrow \mathrm{GP})$ & $\begin{array}{l}\text { Case conference conducted, if } \\
\text { indicated referral to specialist was } \\
\text { recommended }\end{array}$ \\
\hline (3) & $\begin{array}{l}\text { Evaluation by DCM: } \\
\text { Nursing care } \\
\text { necessary? }\end{array}$ & $\begin{array}{l}\text { Baseline and first intervention visit } \\
\text { conducted AND/OR CareNapD: } \\
\text { unmet needs in hygiene AND/OR } \\
\text { dental hygiene AND/OR dressing } \\
\text { AND/OR household AND/OR } \\
\text { health and mobility }\end{array}$ & $\begin{array}{l}\text { Evaluation by DCM: Nursing care } \\
\text { necessary? Recommendation to } \\
\text { GP if indicated }\end{array}$ & $\mathrm{DCM}(\rightarrow \mathrm{GP})$ & $\begin{array}{l}\text { Evaluation conducted, if indicated } \\
\text { nursing care was recommended to } \\
\text { GP }\end{array}$ \\
\hline (4) & $\begin{array}{l}\text { Recommendation to GP: } \\
\text { Indication check } \\
\text { antidementive } \\
\text { medication }\end{array}$ & $\begin{array}{l}\text { ICD diagnosis: dementia AND } \\
\text { medication review: no } \\
\text { antidementive medication }\end{array}$ & $\begin{array}{l}\text { Indication check antidementive } \\
\text { medication }\end{array}$ & $\mathrm{DCM} \rightarrow \mathrm{GP}$ & $\begin{array}{l}\text { Indication check conducted; } \\
\text { antidementive drug prescribed }\end{array}$ \\
\hline (5) & $\begin{array}{l}\text { Recommendation to GP, } \\
\text { delegation of } \\
\text { intervention task to } \\
\text { DCM possible: } \\
\text { Arrange Patient Decree }\end{array}$ & $\begin{array}{l}\text { Severity of dementia: mild cognitive } \\
\text { impairment OR mild dementia } \\
\text { AND no Patient Decree AND } \\
\text { Capability of the patient to grant } \\
\text { consent }\end{array}$ & Arrange Patient Decree & $\begin{array}{l}\mathrm{DCM} \rightarrow \mathrm{GP}(\rightarrow \\
\mathrm{DCM})\end{array}$ & $\begin{array}{l}\text { Recommendation to arrange Patient } \\
\text { Decree given; Patient Decree } \\
\text { arranged }\end{array}$ \\
\hline (6) & $\begin{array}{l}\text { Intervention task for } \\
\text { DCM: Counsel } \\
\text { caregiver regarding } \\
\text { family and partnership }\end{array}$ & $\begin{array}{l}\text { BIZA-D: Family and partnership: } \\
\text { strain } \geq 3\end{array}$ & $\begin{array}{l}\text { Counsel caregiver regarding family } \\
\text { and partnership }\end{array}$ & DCM & Counseling conducted \\
\hline (7) & $\begin{array}{l}\text { Emergency measure: } \\
\text { Suicidal tendency }\end{array}$ & $\begin{array}{l}\text { Manifest suicidal intent/plan } \\
\text { AND/OR suicidal action }\end{array}$ & $\begin{array}{l}\text { Notification of emergency doctor or } \\
\text { GP, stay with proband until help } \\
\text { is ensured }\end{array}$ & DCM & $\begin{array}{l}\text { Notification of emergency doctor or } \\
\text { GP, DCM has put their } \\
\text { instructions into action }\end{array}$ \\
\hline
\end{tabular}

Note: GDS, Geriatric Depression Scale; M.I.N.I., Mini International Neuropsychiatric Interview; NPI, Neuropsychiatric Inventory; CareNapD, Care Needs Assessment Pack for Dementia; ICD-10, International Classification of Disease 10; BIZA-D, Berliner Inventar zur Angehörigenbelastung Demenz; DCM, Dementia Care Manager; DCM $\rightarrow$ GP, recommendation of intervention task to general practioner; $(\rightarrow \mathrm{DCM})$, delegation of intervention task to DCM by GP possible. 
(4) Recommendation to GP: assessment indicated a specific problem that needs to be addressed by GP and/or specialist

(5) Recommendation to GP, delegation of intervention task to DCM possible: assessment indicated a specific problem; the DCM recommends a certain intervention task to the GP who can then delegate this task to the DCM

(6) Intervention task for DCM: assessment indicated an intervention task that the DCM conducts on her own without involving the GP

(7) Emergency measure: in case of emergency the DCM initiates and/or conducts predetermined emergency measures

Even though, in principle, the list of predefined intervention modules forms the basis of DelpHiIntervention, the DCM is not restricted to it. On the one hand the DCM can take additional measures if necessary - as long as these self-assigned tasks comply with principal requirements of the DelpHiIntervention such as (1) not to double already established services but rather integrating the PwD into these or (2) not to violate patient autonomy. On the other hand it is not mandatory to conduct automatically generated intervention tasks if they do not seem appropriate from a clinical and/or nursing perspective. Non-conduction of such tasks needs to be reported and discussed in a case conference (see below). Reasons for adding as well as removing intervention tasks are documented.

Full documentation of both initiation and completion (including reasons for non-completion, if applicable) of all triggered as well as self-assigned intervention tasks is a vital part of the DelpHi-study.

A computer-assisted Intervention-ManagementSystem (IMS) was developed to facilitate the planning and documentation of the DelpHiIntervention. Rules for all predefined intervention modules were programmed. For data collection, the DCM uses a hand-held tablet computer with touch-screen functionality. All information entered by the DCM into the central DelpHi-information system is processed immediately (Meyer et al., 2012). Whenever a predefined trigger condition is activated, the corresponding intervention task is automatically selected and assigned, including tasks for in-depth assessments and case conferences.

The DCM conducts all in-depth assessments indicated by the IMS (e.g. mobility tests, pain assessment, neuropsychiatric assessment) usually in her third home visit with the PwD. Based on these data, the IMS generates an individual preliminary list for the respective subject with suggestions for specific intervention tasks. This list is discussed and finalized in a weekly interdisciplinary case conference. Participants of this conference are the DCM, a nursing scientist, a neurologist/psychiatrist, a psychologist, and a pharmacist. The case conference is an important part of the study to check adherence to the study protocol as well as to assess the adequateness of the predefined intervention modules. Hence the case conference provides crucial information for later evaluation of the DelpHi-Intervention.

After the case conference the main results of the baseline and in-depth assessment as well as the finalized list of intervention tasks are summarized by the DCM in a semi-standardized GP- information letter that contains (1) a cover letter that gives a brief description of the patient's social, physical, and mental needs and resources as well as some prioritized suggestions for intervention, (2) a standardized report of the most important outcomes of baseline and in-depth assessments, and (3) a list of recommended intervention tasks. This letter is then discussed between GP and DCM to establish an individual treatment plan. Finally the GP decides whether s/he will conduct a suggested intervention task, delegate a task to the DCM, or rate a task as "not necessary."

The GP-information letter is the main instrument for communication and coordination of DCM and GP and ensures that the GP plays a key role in the context of the DelpHi-Intervention.

During the first six months of the intervention period, the DCM conducts home visits at least monthly, taking all necessary steps to carry out her designated intervention tasks in close cooperation with the caregiver, the GP, and various healthcare and social service professionals and facilities.

The subsequent six months the DCM monitors the completion of all intervention tasks, mainly by telephone, but also in additional home visits if necessary.

\section{Follow-up assessment}

The intervention period is completed with the follow-up assessment after one year, which is conducted in the control group as well. To assess long-term effects of the intervention, annual followup assessments will be conducted in both groups for at least five years.

\section{Expected results and discussion}

We expect at least small effects of the DelpHiIntervention, i.e. statistically significant differences between control and intervention groups across the primary outcomes. In concrete terms, we expect for the intervention group (1) better quality of life of the PwDs and their caregivers, (2) less behavioral and psychological symptoms of dementia, (3) more frequent medical treatment with 
antidementive drugs and less frequent potentially inadequate medication, and (4) less caregiver burden as in the control group. Comprehensive economic analyses will be conducted to determine the cost effectiveness of the DelpHi-Intervention, a prerequisite for the translation of DelpHiIntervention into routine healthcare. For details and further information on intended statistical analyses, see the study protocol (Thyrian et al., 2012).

The Delphi-Intervention meets two requirements for the effectiveness of collaborative care models (Somme et al., 2012): (1) high intensity of care management, and (2) high level of integration. Pacala et al. (1995) defined a scale to rate case management intensity based on 18 different criteria. Only programs with high intensity of intervention and at least medium integration level reported moderate to medium clinical effects on their primary outcomes (Callahan et al., 2006; Vickrey et al., 2006), whereas low-intensity programs reported none or slight but clinically insignificant effects (Weinberger et al., 1993; Yordi et al., 1997; Bass et al., 2003). The DelpHi-Intervention meets 14 of Pacala's criteria and can be classified as "high intensity" case management accordingly. Integration level can be rated "high" as well: the DCMs cooperate closely with caregivers, GPs, and various healthcare and social service professionals and facilities to integrate PwDs into the established healthcare and social service system.

With the Delphi-Intervention we want to provide "optimum care" to PwDs and their caregivers. The intervention is comprehensive, integrative, multi-professional, multimodal, individualized, and therefore highly complex. To facilitate the handling of this complexity, the computer-assisted IMS was developed. To our knowledge, DelpHi-MV is the first randomized controlled intervention trial to utilize a comprehensive computer-assisted management system for ambulant dementia care management. The deployment of IMS has several benefits. It helps to ensure that all individual needs and resources of the PwDs and their caregivers as well as all necessary intervention tasks according to the "DelpHi-Standard" are considered by the DCM. However, IMS is merely an instrument to support the work of the DCM. In collaboration with the interdisciplinary case conference, the DCM has to assess the adequateness of each intervention task generated by IMS as well as to decide if additional interventions are required. Hence the IMS does neither take any decision nor provides diagnosis or therapeutical advice; thus it complies to legal requirements of the German Medical Products Law (MPG; Bundesministerium der Justiz, 1994).

Besides IMS supports adherence of the DCM to the study protocol, thereby enhancing the quality of implementation of the DelpHi-Intervention. IMS is also a valuable tool for the evaluation of the DelpHi-Intervention. It enables the analysis of research questions such as: (1) which intervention tasks were generated under which conditions, (2) what were the most frequently generated intervention tasks, (3) what are the reasons for non-conduction of intervention tasks, (4) which interventions had an impact on primary outcomes, (5) which intervention tasks were delegated by GP to the DCM, (6) which intervention tasks were successfully conducted, and (7) which intervention tasks were rated as not necessary by the GP. Results of these analyses will not only provide answers to these questions but will also be used for refinements of DelpHi-Intervention by adding missing intervention modules and adapting inappropriate trigger conditions and/or subsequent intervention tasks.

Results of this study can provide evidence for the effectiveness and efficacy of collaborative dementia care management according to the "Delphi-Standard," paving the way for translating this innovative concept into routine healthcare.

\section{Conflict of interests}

None.

\section{Description of authors' roles}

All authors contributed to the development of the DelpHi-Intervention in their area of special expertise. TE drafted the paper and was a main contributor to the development of the IMS. JRT is the coordinator of the study and has substantially contributed to the overall design of the study as well as the development of DelpHi-Intervention. $\mathrm{AD}$ was responsible for qualification of DCM and the planning of the action field "nursing care." DW and TF were responsible for the pharmaceutical, GB for the neuropsychiatric part of intervention. LK is responsible for data bank management and $\mathrm{BM}$ for health economic analyses. WH is the principal investigator of the study and has contributed substantially to the concept of DelpHiMV. All authors read and approved the final paper.

\section{Acknowledgments}

The development and establishment of the DelpHiIntervention was influenced by input from several experts in their respective field and supported by an experienced field study team. These are namely 
(in alphabetical order): Kerstin Albuerne, Aniela Angelow, Grit Aßmann, Vaska Böhmann, Kathleen Dittmer, Daniel Fredrich, Sarah Gardzella, Jana Hubert, Ulrike Kempe, Ingo Kiliman, Saskia Moll, Christiane Schnick, Stefan Teipel, Christine Winckler, and Paula Winter

\section{References}

Basler, H. D. et al. (2006). Beurteilung von Schmerz bei Demenz (BESD). Untersuchung zu Validität eines Verfahrens zur Beobachtung des Schmerzverhaltens. Der Schmerz, 20, 519-526.

Bass, D. M., Clark, P. A., Looman, W. J., McCarthy, C. A. and Eckert, S. (2003). The Cleveland Alzheimer's managed care demonstration: outcomes after 12 months of implementation. Gerontologist, 43, 73-85.

Berg, L. (1988). Clinical Dementia Rating (CDR). Psychopharmacology Bulletin, 24, 637-639.

Black, W. and Almeida, O. P. (2004). A systematic review of the association between the Behavioral and Psychological Symptoms of Dementia and burden of care. International Psychogeriatrics, 16, 295-315.

Bullinger, M. and Kirchberger, I. (1998). SF-36 Fragebogen zum Gesundheitszustand. Handanweisung. Göttingen: Hogrefe Verlag für Psychologie.

Bundesministerium der Justiz (1994). Gesetz über Medizinprodukte (Medizinproduktegesetz-MPG). Available at: http://www.gesetze-im-internet.de/bundesrecht/mpg/ gesamt.pdf; last accessed 7 March 2013.

Calabrese, P. and Kessler, J. (2000). Screening for cognitive impairment in dementia - the DemTect procedure. European Neuropsychopharmacology, 10, 369.

Callahan, C. M. et al. (2006). Effectiveness of collaborative care for older adults with Alzheimer disease in primary care: a randomized controlled trial. $\mathcal{F} A M A, 295,2148-2157$.

Cummings, J. L. (1997). The Neuropsychiatric Inventory: assessing psychopathology in dementia patients. Neurology, 48, 10-16.

DEGAM (Deutsche Gesellschaft für Allgemeinmedizin und Familienmedizin e.V.) (2008). DEGAM-Leitlinie $\mathrm{Nr}$ 12: Demenz. Düsseldorf: omikron.

Derogatis, L. R. and Melisaratos, N. (1983). The Brief Symptom Inventory: an introductory report. Psychological Medicine, 13, 595-605.

DGPPN and DGN (Deutsche Gesellschaft für Psychiatrie, Psychotherapie und Nervenheilkunde and Deutsche Gesellschaft für Neurologie (2009). S-3 Leitlinie “Demenzen.” Available at: http://www.dgppn.de/ fileadmin/user_upload/_medien/download/pdf/ kurzversion-leitlinien/s3-leitlinie-demenz-lf.pdf; last accessed 7 March 2013.

Dreier, A., Thyrian, J. R. and Hoffmann, W. (2011). Dementia care manager in der ambulanten demenzversorgung: entwicklung einer innovativen qualifizierung für pflegefachkräfte. Pflege $\mathcal{E}$ Gesellschaft, 16, 53-64.

Etters, L., Goodall, D. and Harrison, B. E. (2008). Caregiver burden among dementia patient caregivers: a review of the literature. Fournal of American Academy of Nurse Practitioners, 20, 423-428.

Fendrich, K. and Hoffmann, W. (2007). More than just aging societies: the demographic change has an impact on actual numbers of patients. Fournal of Public Health, 15, 345-351.

Fiß, T., Thyrian, J. R. and Hoffmann, W. (2013). Medication management for people with dementia in primary care: description of implementation in the DelpHi-study. BMC Geriatrics (submitted).

Förstl, H. (2009). Demenzen in Theorie und Praxis. Heidelberg: Springer Medizin Verlag.

Fydrich, T., Sommer, G. and Brähler, E. (2007). F-SozU. Fragebogen zur sozialen Unterstützung. Göttingen: Hogrefe.

Hampel, H. et al. (2011). The future of Alzheimer's disease: the next 10 years. Progress in Neurobiology, 95, 718-728.

Hindmarch, I., Lehfeld, H., de Jongh, P. and Erzigkeit, H. (1998). The Bayer Activities of Daily Living Scale (B-ADL). Dementia and Geriatric Cognitive Disorder, 9, 20-26.

Holt, S., Schmiedl, S. and Thürann, P. A. (2010). Potentially inappropriate medications in the elderly: the PRISCUS list. Deutsches Ärtzeblatt International, 107, 543-551.

Kessler, J., Markowitsch, H. J. and Denzler, P. (1990). Mini-Mental-Status-Test (MMST) [German Version]. Göttingen: Beltz Test GmbH.

Lecrubier, Y. et al. (1997). The Mini International Neuropsychiatric Interview (MINI). A short diagnostic structured interview: reliability and validity according to the CIDI. European Psychiatry, 12, 224-231.

Logsdon, R. G., Gibbons, L. E., McCurry, S. M. and Teri, L. (2002). Assessing quality of life in older adults with cognitive impairment. Psychosomatic Medicine, 64, 510-519.

Löwe, B., Zipfel, S. and Herzog, W. (2002). PHQ-D Gesundheitsfragebogen für Patienten. New York: Pfizer.

McWalter, G., Toner, H., McWalter, A., Eastwood, J., Marshall, M. and Turvey, T. (1998). A community needs assessment: the care needs assessment pack for dementia (CarenapD) - its development, reliability and validity. International fournal of Geriatric Psychiatry, 13, 16-22.

Meyer, J., Fredrich, D., Piegsa, J., Habes, M., van den Berg, N. and Hoffmann, W. (2012). A mobile and asynchronous electronic data capture system for epidemiologic studies. Computer Methods and Programs in Biomedicine, 107, 425-435.

Mittelman, M. S., Haley, W. E., Clay, O. J. and Roth, D. L. (2006). Improving caregiver well-being delays nursing home placement of patients with Alzheimer disease. Neurology, 67, 1592-1599.

Monahan, P. O. et al. (2012). Practical clinical tool to monitor dementia symptoms: the HABC-Monitor. Fournal of Clinical Interventions in Aging, 7, 143-157.

Naylor, M. D. et al. (2012). Advancing Alzheimer's disease diagnosis, treatment, and care: recommendations from the Ware Invitational Summit. Alzheimer's $\mathcal{E}$ Dementia, 8, 445-452.

Pacala, J. T. et al. (1995). Case management of older adults in health maintenance organizations. Fournal of the American Geriatric Society, 43, 538-542. 
Podsiadlo, D. and Richardson, S. (1991). The timed "Up \& Go": a test of basic functional mobility for frail elderly persons. Fournal of the American Geriatric Society, 39, 142-148.

Reisberg, B., Ferris, S. H., de Leon, M. J. and Crook, T. (1988). Global Deterioration Scale (GDS). Psychopharmacology Bulletin, 24, 661-663.

Reuben, D. et al. (2009). Closing the dementia care gap: can referral to Alzheimer's Association chapters help? Alzheimer's E Dementia, 5, 498-502.

Sandholzer, H., Hellenbrand, W., Renteln-Kruse, W., van Weel, C. and Walker, P. (2004). STEP standardized assessment of elderly people in primary care. Deutsche Medizinische Wochenschrift, 129, S183-S226.

Somme, D., Trouve, H., Drame, M., Gagnon, D., Couturier, Y. and Saint-Jean, O. (2012). Analysis of case management programs for patients with dementia: a systematic review. Alzheimer's $\mathcal{E}$ Dementia, 8, 426-436.

Thyrian, J. R. et al. (2012). Dementia: life- and person-centered help in Mecklenburg-Western Pomerania, Germany (DelpHi) - study protocol for a randomised controlled trial. Trials, 13, 56.

Tinetti, M. E. (1986). Performance-oriented assessment of mobility problems in elderly patients. Fournal of the American Geriatric Society, 34, 119-126.

Vickrey, B. G. et al. (2006). The effect of a disease management intervention on quality and outcomes of dementia care: a randomized, controlled trial. Annals of Internal Medicine, 145, 713-726.

Vollmar, H., Koch, M., Löscher, S. and Butzlaff, M. (2007). Leitlinie Diagnose, Therapie und Versorgung bei Demenz Version 05/05. Available at: http://www.evidence.
de/Leitlinien/leitlinien-intern/Demenz_Start/demenz_start. html; last accessed 7 March 2013.

Wagner, G. and Abholz, H. (2002). Diagnose und Therapiemanagement der Demenz in der Hausarztpraxis. Zeitschrift für Allgemeinmedizin, 78, 239-244.

Weinberger, M. et al. (1993). Social service interventions for caregivers of patients with dementia: impact on health care utilization and expenditures. Fournal of the American Geriatric Society, 41, 153-156.

Wimo, A., Wetterholm, A., Mastey, V. and Winbald, B. (1998). Evaluation of resource utilization and caregiver time in anti-dementia drug trials: a quantitative battery. In A. Wimo, B. Jonsson, G. Karlson and B. Winbald (eds.), The Health Economics of Dementia (pp. 465-499). London: John Wiley.

World Health Organization (WHO) (1986). Ottawa-Charta zur Gesundheitsförderung. Available at: http://www.euro. who.int/_data/assets/pdf_file/0004/129532/Ottawa_ Charter.pdf; last accessed 7 March 2013.

Yordi, C., DuNah, R., Bostrom, A., Fox, P., Wilkinson, A. and Newcomer, R. (1997). Caregiver supports: outcomes from the Medicare Alzheimer's disease demonstration. Health Care Financing Review, 19, 97-117.

Zank, S., Schacke, C. and Leipold, B. (2006). Berliner Inventar zur Angehörigenbelastung - Demenz (BIZA-D). Zeitschrift für Klinische Psychologie und Psychotherapie, 35, 296-305.

Zaudig, M. et al. (1991). SIDAM: a structured interview for the diagnosis of dementia of the Alzheimer type, multi-infarct dementia and dementias of other aetiology according to ICD-10 and DSM-III-R. Psychological Medicine, 21, 225-236. 